First publ. in: Physics Letters B 300 (1993), 3, pp. 210-216

\title{
Improved limit on the electron-antineutrino rest mass from tritium $\beta$-decay
}

\author{
Ch. Weinheimer, M. Przyrembel , H. Backe, H. Barth, J. Bonn, B. Degen, Th. Edling, \\ H. Fischer, L. Fleischmann, J.U. Grooß, R. Haid ; A. Hermanni, G. Kube, P. Leiderer ${ }^{1}$, \\ Th. Loeken, A. Molz, R.B. Moore ${ }^{2}$, A. Osipowicz, E.W. Otten, A. Picard, M. Schrader \\ and M. Steininger
}

Institut für Physik, Universität Mainz, W-6500 Mainz, FRG

Received 4 November 1992

The endpoint region of the $\beta$-spectrum of tritium was remeasured by an electrostatic spectrometer with magnetic guiding field. It enabled the search for a rest mass of the electron-antineutrino with improved precision. The result is $m_{\nu}^{2}=-39 \pm 34_{\text {stat }} \pm 15_{\text {syst }}\left(\mathrm{eV} / \mathrm{c}^{2}\right)^{2}$, from which an upper limit of $m_{\nu}<7.2 \mathrm{eV} / \mathrm{c}^{2}$ may be derived. The experiment yields the atomic mass difference $m(\mathrm{~T})-m\left({ }^{3} \mathrm{He}\right)=18591 \pm 3 \mathrm{eV} / \mathrm{c}^{2}$.

The question of a non-vanishing neutrino rest mass is of fundamental importance in particle and astrophysics. It therefore caused considerable excitement when Lubimov and collaborators [1,2] published a finite value for the electron-antineutrino rest mass $m_{\nu}$ obtained from a measurement of the endpoint region of the $\beta$-decay of tritium with a new, dedicated spectrometer of high resolution and luminosity [3]. It took a number of years until this result was checked with equivalent instruments by groups in Zürich [4] and Los Alamos [5] and disproved due to essential improvements of source and data analysis $[6,7]$. At present, it seems that this generation of experiments is capable of reaching a precision of $\Delta m_{\nu}^{2} \approx 100\left(\mathrm{eV} / \mathrm{c}^{2}\right)^{2}$ (see table 3$)$.

A radically different type of $\beta$-spectrometer was proposed independently at several places $[8,9]$. It acts as an electrostatic filter guiding the electrons adiabatically along the lines of an inhomogeneous magnetic field. If the source is placed in a strong magnetic field $B_{0}$ and the retarding potential $U_{0}$ reaches its maximum in a much weaker magnetic field $B_{1}$, the full forward solid angle of electrons emitted with energy

1 Present address: Fakultät für Physik, Universität Konstanz, W-7750 Constance, FRG.

2 Present address: McGill University, Montreal, Canada.
$E$ is analyzed with a filter width of $\Delta E=\left(B_{1} / B_{0}\right) E$. This is due to the adiabatic transformation of transverse cyclotron energy $E_{\perp}$ around the B-lines into longitudinal energy $E_{\|}$along the $B$-lines by the magnetic field gradient reducing $E_{\perp}$ (which cannot be analyzed electrostatically) in proportion to the magnetic field. Based on this principle, two somewhat different spectrometers have been set up in recent years at INR in Troitzk [10] and at Mainz University. For details of the design, function and performance of the Mainz solenoid retarding spectrometer (SRS) and for further references see refs. $[11,12]$.

The experiment described here was performed under the following conditions. The source is placed at a field $B_{\mathrm{S}}=0.96 B_{0}$, slightly in front of the field maximum of the source solenoid which is set to $B_{0}=2.4$ $\mathrm{T}$, limiting the accepted polar angles to $\vartheta<78^{\circ}$. The magnetic field reaches its minimum $B_{1}=8 \times 10^{-4}$ $\mathrm{T}$ in the symmetry plane of the spectrometer, where $U_{0}$ maximizes. Retardation of the electrons and reacceleration after the filter is provided by two sets of electrodes arranged symmetrically around the central one. Under these conditions the rise of the transmission from 0 to 1 within the interval $E\left(1-B_{1} / B_{0}\right) \leqslant$ $e U_{d} \leqslant E$ is given by [12] 


$$
\begin{aligned}
& T\left(E, U_{\mathrm{d}}\right) \\
& =1-\sqrt{1-\frac{E-e U_{\mathrm{d}}}{E} \frac{B_{\mathrm{S}}}{B_{1}}}\left(1-\sqrt{1-\frac{B_{\mathrm{S}}}{B_{0}}}\right)^{-1}
\end{aligned}
$$

where $U_{\mathrm{d}}=U_{\mathrm{S}}-U_{0}$ is the difference between the potentials of the source and the central electrode. $T\left(E, U_{\mathrm{d}}\right)$ was checked with high accuracy by conversion electrons from ${ }^{83 \mathrm{~m}} \mathrm{Kr}$ [12]. As important as the sharpness of the filter is the absence of any tails of $T\left(E, U_{\mathrm{d}}\right)$ extending beyond $E$. During reacceleration, the electrons are also refocussed by the field of a second solenoid, also set to $2.4 \mathrm{~T}$, and finally reach a silicon detector placed in the central field $B_{\mathrm{D}}=0.8 \mathrm{~T}$ of a third solenoid. The active area of the detector has a diameter of $25 \mathrm{~mm}$ and is segmented into five rings of equal area. The counts were pulse height analyzed and stored event by 'event. Cooled down to $-80^{\circ} \mathrm{C}$, the detector has a resolution of $2.0 \mathrm{keV} \mathrm{FWHM} \mathrm{for}$ $20 \mathrm{keV}$ electrons. The resolution was somewhat degraded with respect to the values reported in ref. [13] due to $15 \mu \mathrm{g} / \mathrm{cm}^{2}$ aluminium evaporated onto the $30 \mu \mathrm{g} / \mathrm{cm}^{2}$ Kapton foil separating the high vacuum at the detector from the UHV in the spectrometer.

Regarding the source, we decided on molecular $\mathrm{T}_{2}$ frozen onto an aluminium substrate cooled down to $2.8 \mathrm{~K}$. Compared to any other T-compound, this choice offers the highest specific activity. Because of the lowest possible $Z$, the spectrum of energy losses by inelastic scattering within the source, as well as by prompt shake up/off processes, is, in comparison, also soft and simple. According to extensive molecular orbit calculations [14], the final state spectrum of the latter is slightly more complicated than that of gaseous $T_{2}$ [15]. The present source was constructed following the experience of a feasibility study [16]. The substrate is mounted on the front of a $1.2 \mathrm{~m}$ long, horizontal LHe cryostat. The solid angle of $T_{2}$-evaporation into the spectrometer is limited to $\Delta \Omega / 4 \pi=2.5 \times 10^{-3}$ by a LHe-cooled, $10 \mathrm{~cm}$ long and $2 \mathrm{~cm}$ wide $\mathrm{Cu}$ tube in front of the source which itself covers a circular area of $1 \mathrm{~cm}^{2}$. The tube also reduces condensation of residual gas onto the source. The source is connected to the spectrometer by a bellows allowing it to be moved from the loading to the measuring position through a valve. $T_{2}$ is evaporated onto the substrate by covering the respective area with a teflon cup into which $T_{2}$ is led through a capillary. Glass windows allow the evaporation process to be controlled by ellipsometry. Films of 40 monolayers, corresponding to a total source strength of $10^{8} \mathrm{~Bq}$ were prepared. Through on line mass spectrometry we detected tritium contaminations of about $30 \%$ of hydrogen which had probably taken place in the stainless steel container. The source region meets the UHV conditions of the spectrometer [11]. During measurements the source "decayed" almost exponentially with a half-life time of a week. Data were taken for about ten days persource.

Without a source the background spectrum peaked at about $23 \mathrm{keV}$, well above the tritium spectrum [11]. Therefore, most of it could be suppressed by limiting the window of accepted events between 12 and 19.5 $\mathrm{keV}$. The residual background rate then dropped to $5 \mathrm{mHz}$ for the central segment and to $23 \mathrm{mHz}$ for the outermost one. With the tritium source the background rate rose by a factor of up to 2 for a fresh source. This additional background peaked at the energy $-e U_{0}$. A rough estimation showed that it could be attributed to $T_{2}$ molecules which evaporate from the source and decay in vacuo within the magnetic flux tube projected onto the detector. After removing the source the background rate returned immediately to the original value showing no obvious sign of contamination of the spectrometer.

Tritium spectra were recorded in the energy interval $18095 \leqslant e U_{\mathrm{d}} \leqslant 18800 \mathrm{eV}$ by scanning up and down a negative potential $U_{\mathrm{S}}$ on the source ${ }^{\# 1}$ at constant analyzing potential $U_{0}=-18779 \mathrm{~V}$. The most critical region around the endpoint was scanned in steps of $4 \mathrm{~V}$ with an integration time of $2 \cdot 30 \mathrm{~s}$ per point and scan. Elsewhere larger steps and shorter integration times were chosen. The data were screened for false events. By checking the distribution of time differences between events we detected sudden increases in the count rate, possibly triggered by microsparks in the spectrometer. About $14 \%$ of the whole set of about 500 scans have been rejected due to this failure. The scatter of the remaining data obeys a statistical distribution.

\#1 Negative source potential is essential for retaining ions from $T_{2}$ decay which are otherwise accelerated into the spectrometer causing a few $\mathrm{Hz}$ background rate by ionization of residual gas. 


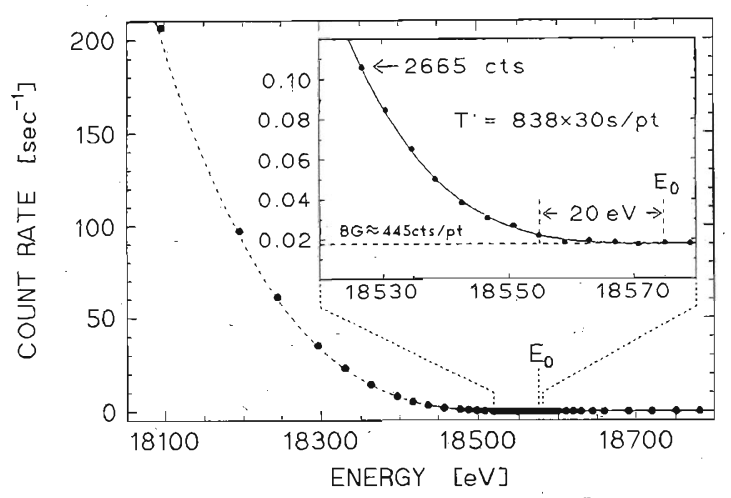

Fig. 1. $\beta$-spectrum of tritium recorded during a four-week run in 1991. The statistical error bars are too small to be plotted. For energies $E_{e} \geqslant 18460 \mathrm{eV}$ the integral measuring time is $25140 \mathrm{~s}$ per point. The background level is $450 \mathrm{cts}$ per point $\widehat{=} 18 \mathrm{mHz}$. The full line is the best fit to the data in the interval from 18438 to $18800 \mathrm{eV}$, the broken one is the extrapolation of the fit to lower energies.

Fig. 1 shows the recorded $\beta$-spectrum. The data comprise counts of the two.innermost segments of the detector. The other ones are covered only partly by the image of the source and suffer from higher background. An expanded view of the endpoint region is given in the insert. Already $20 \mathrm{eV}$ below the endpoint, the spectrum emerges clearly from the background noise.

Another instructive view to the data is obtained from a linearized plot of the spectrum given in fig. 2 . Since our spectrometer is integrating the $\beta$-spectrum, the linearization is achieved to a first approximation by the cube root of the count rate after substracting the background. The data deviate from the linear slope as soon as transitions to exited states of $\left({ }^{3} \mathrm{HeT}\right)^{+}$become significant. The straight line representing transitions to the ground state of $\left({ }^{3} \mathrm{He} \mathrm{T}\right){ }^{+}$intersects the baseline about $4 \mathrm{eV}$ below the endpoint. This is mainly due to the average residual energy in the motion of the electrons around their guiding field lines which is not analyzed by the SRS. The fit, described below, on the other hand slightly. overshoots the endpoint, as the best fit value for $m_{\nu}^{2}$ is negative. Furthermore, we have plotted into fig. 2 fits to the data with $m_{\nu}$ fixed to 0,10 and $20 \mathrm{eV} / c^{2}$ respectively. A value of the order of $20 \mathrm{eV} / \mathrm{c}^{2}$ is excluded apparently. As to our knowledge, it is the first time that such fine details have ever been resolved in a $\beta$-spectrum.

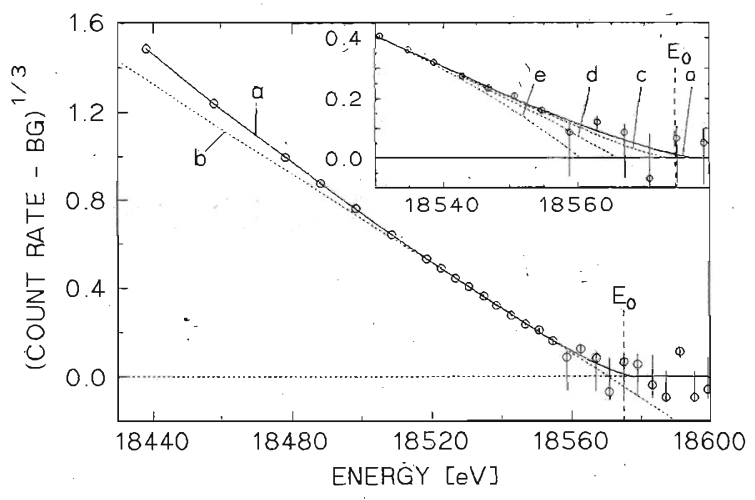

Fig. 2. Linearized $\beta$-spectrum close to the endpoint. Curve a: best fit with $m_{\nu}^{2} c^{4}=-39 \pm 34(\mathrm{eV})^{2}$ and experimental $\beta$-endpoint $E_{0}=18574.8 \pm 0.6 \mathrm{eV}$; curve b: linear fit of last $50 \mathrm{eV}$; curves c-e: fits with $m_{\nu} c^{2}$ fixed to $0,10,20 \mathrm{eV}$ in the interval $18438-18600 \mathrm{eV}$.

In the final evaluation the data were fitted to the sum of a background function

$b\left(U_{\mathrm{d}}\right)=b_{0}+b_{1}\left(E_{0}-e U_{\mathrm{d}}\right)$

and a convolution

$$
\begin{aligned}
& I\left(U_{\mathrm{d}}\right)=\iint T\left(E^{\prime}, U_{\mathrm{d}}\right) D\left(E^{\prime}, U_{\mathrm{d}}\right) \\
& \quad \times L\left(E, E^{\prime}\right) S(E) \mathrm{d} E \mathrm{~d} E^{\prime}
\end{aligned}
$$

of the transmission function of the spectrometer $T\left(E^{\prime}, U_{\mathrm{d}}\right)$, eq. (1), the detector efficiency function $D\left(E^{\prime}, U_{\mathrm{d}}\right)=1+\alpha_{\mathrm{D}}\left(E^{\prime}-e U_{\mathrm{d}}\right)$ with $\alpha_{\mathrm{D}}=0.10 \pm 0.01$ $\mathrm{keV}^{-1}$, the energy loss function $L\left(E, E^{\prime}\right)$ and the spectral function of the $\beta$-decay $S(E)$.

The background is entirely determined by the data measured beyond $E_{0}$ yielding $b_{0}=17.7(2) \mathrm{mHz}$, $b_{1}=5(3) \mu \mathrm{Hz} / \mathrm{V}$.

The energy loss function of the sources $L\left(E, E^{\prime}\right)$ has been calculated from an inelastic cross section which is approximated by

$$
\begin{aligned}
& \frac{\mathrm{d} \sigma\left(E, E^{\prime}\right)}{\mathrm{d} E^{\prime}}=\frac{a_{\mathrm{exc}} \Gamma_{\mathrm{exc}}^{2}}{\Gamma_{\mathrm{exc}}^{2}+\left(\Delta E-E_{\mathrm{exc}}\right)^{2}} \\
& \times \Theta\left(\Delta E-E_{\mathrm{min}}\right) \Theta\left(E_{\mathrm{B}}-\Delta E\right) \\
& +\frac{a_{\text {ion }} \Gamma_{\mathrm{ion}}^{2}}{\Gamma_{\mathrm{ion}}^{2}+\left(\Delta E-E_{\mathrm{ion}}\right)^{2}} \Theta\left(\Delta E-E_{\mathrm{B}}\right)
\end{aligned}
$$

with $\Delta E=E-E^{\prime}, E_{\min }=8.8 \mathrm{eV}, E_{\mathrm{B}}=15.4$ $\mathrm{eV}, a_{\mathrm{exc}}=7.5 \times 10^{-19} \mathrm{~cm}^{2} /(\mathrm{eV}$ molecule $), \Gamma_{\mathrm{exc}}=$ 
$0.8 \mathrm{eV}, E_{\text {exc }}=12.6 \mathrm{eV}, a_{\text {ion }}=1.5 \times 10^{-19} \mathrm{~cm}^{2} /$ (eV molecule), $\Gamma_{\text {ion }}=7.1 \mathrm{eV}, E_{\text {ion }}=17.2 \mathrm{eV}$.

The first lorentzian approximates the excitation of $T_{2}$ [17], the second the ionisation [18]. The parameters $a_{\mathrm{exc}}$ and $a_{\text {ion }}$ are chosen to match the total stopping power and the total inelastic cross section $[19,20]$. The zero loss fraction of electrons is $(91 \pm 4) \%$ in the average calculated from eq. (4). The error is dominated by uncertainties in the tritium film thickness and homogeneity.

The spectrum is described by

$$
\begin{aligned}
& S(E)=A F p\left(E+m_{e} c^{2}\right) \\
& \quad \times \sum_{i} W_{i} \varepsilon_{i} \sqrt{\varepsilon_{i}^{2}-m_{\nu}^{2} c^{4}}\left(1+\frac{1}{3} \alpha_{\mathrm{BS}} \varepsilon_{i}\right)
\end{aligned}
$$

with $A$ is the amplitude, $F$ is the Fermi function [21], $p$ is the electron momentum, $\varepsilon_{i}=E_{0}-V_{i}-E, W_{i}$ is the relative transition probability to the $i$ th molecular final state of excitation energy $V_{i}$. The backscatter contribution is convoluted with the spectrum in linear approximation by the last factor in eq. (5) with $\alpha_{\mathrm{BS}}=0.20 \pm 0.05 \mathrm{keV}^{-1}$, which was derived from preliminary test measurements. To enable fitting around $m_{\nu}^{2}=0$ we use a continuation of the term $\varepsilon_{i} \sqrt{\varepsilon_{i}^{2}-m_{\nu}^{2} c^{4}}$ into the region $m_{\nu}^{2}<0$ replacing it by $\varepsilon_{i}+\mu \exp \left(-\varepsilon_{i} / \mu-1\right) \sqrt{\varepsilon_{i}^{2}-m_{\nu}^{2} c^{4}}$ with $\mu=$ $0.76 \sqrt{-m_{\nu}^{2} c^{4}}$. This continuation is smooth and provides a parabolic $\chi^{2}$-distribution around $m_{\nu}^{2}=0$. To save computing time we have replaced the sum over the final states which comprises the product of the final state spectrum of the daughter molecule $\left({ }^{3} \mathrm{HeT}\right)^{+}$ [15] and the simultaneously excited closest neighbours [14] by 10 discrete states with appropriated gaussian widths. This procedure has been checked to be sufficiently precise.

The free fit parameters are $A, E_{0}, m_{\nu}^{2}, b_{0}$ and $b_{1}$. Fig. 3 shows the best fit results for $m_{\nu}^{2}, E_{0}$ and $\chi^{2} /$ DOF as a function of the lower limit $E_{l}$ of the fit interval. The significant dependence on this boundary points to residual, systematic errors correlating to $m_{\nu}^{2}$ and $E_{0}$ further below the endpoint: Although being. small they may $\operatorname{drag} m_{\nu}^{2}$ and $E_{0}$ away from the true values because the statistical weight of data points increases rapidly with decreasing energy. As shown by the conservative systematic errors deduced for some of the $m_{\nu}$ values shown in fig. 3 , we believe

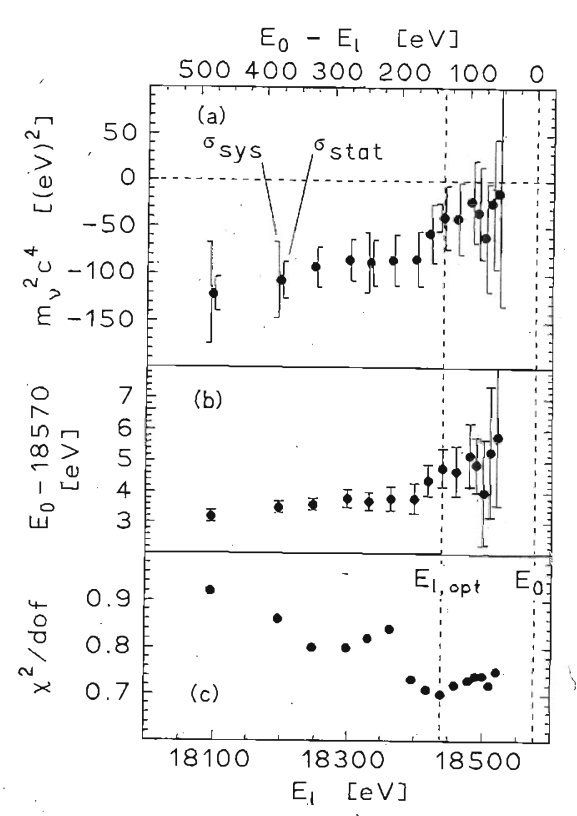

Fig. 3. (a) Square of neutrino rest mass $m_{\nu}^{2}$, (b) endpoint $E_{0}$ and (c) $\chi^{2} / \mathrm{DOF}$ as function of the lower limit $E_{l}$ of the fit interval.

it unlikely that uncertainties in energy loss, backscatter, spectrometer function etc. could be ressponsible for this unphysical trend. For $E_{l}=E_{0}-137 \mathrm{eV}$ this sýstematic error is broken down into its components in table 1. The systematic uncertainties about the final states distribution were checked by using an alternative calculation $[22,23]$. The results were essentially unchanged. The trend to negative $m_{\nu}^{2}$ arises from an excess count rate far from the endpoint. A simple way to account for this would be to increase the shake off probability. To test this, we changed the shake off probability from $15 \%$ as given in ref. [15] over a wide range ${ }^{\# 2}$. Át $21 \%$ all fit parameters, as well as $\chi^{2} / \mathrm{DOF}$, remain stable ágainst variation of the fit interval with $m_{\nu}^{2}$ compatible to zero within $1 \sigma$. statistical error ${ }^{\# 3}$.

The high resolution and statistics together with the low background of our experiment allow for the first time to reduce the problems associated with inciuding

\#2 Some evidence also seems to exist that shake off probabilities measured in conversion electron spectra [24] exceed calculated ones [25].

\#3 For footnote 3 see next page. 
Table 1

Influence of variation of critical parameters on $m_{\nu}^{2}$ at $E_{l, \mathrm{opt}}=E_{0}-137 \mathrm{eV}$. The coefficients $\alpha_{D}$ for detector efficiency and $\alpha_{\mathrm{BS}}$ for backscatter were changed simultanously since they have the same influence on $I\left(U_{\mathrm{d}}\right)$, despite of their different functional dependence. The constant backscatter spectrum was changed by allowing a strong additional linear term keeping the total backscattering probability constant over the interval where the backscatter distribution was investigated. The fraction of energy loss by excitation was changed by $50 \%$ while keeping the total inelastic cross section constant. This accounts for different stopping power values given in literature.

\begin{tabular}{lcc}
\hline Parameter & Change $(\%)$ & $\Delta m_{\nu}^{2} c^{4}\left((\mathrm{eV})^{2}\right)$ \\
\hline inelastic scattering & 50 & \\
total probability & 50 & 14.2 \\
$a_{\text {exc }}\left(\sigma_{\text {tot }}=\right.$ const. $)$ & 25 & 3.3 \\
backscatter and detector efficiency & - & 1.8 \\
$\alpha_{\mathrm{BS}}, \alpha_{\mathrm{D}}$ & 10 & 3.2 \\
different shape & - & 1.4 \\
width of $\dot{T}\left(E^{\prime}, U_{\mathrm{d}}\right)$ & & 0.3 \\
alternative final state distribution & a) \\
total & & 15.1 \\
\hline
\end{tabular}

a) Refs. [22,23].

-a wider range of the $\beta$-spectrum into the data evaluation. We therefore chose $E_{l, \mathrm{opt}}=E_{0}-137 \mathrm{eV}$, where the fraction of ground state transitions is $76 \% \# 4$. The data were fitted for the two sources separately and combined (see table 2). As a final result we obtain from this interval:

$m_{\nu}^{2} c^{4}=-39 \pm 34_{\text {stat }} \pm 15_{\text {syst }}(\mathrm{eV})^{2}$

and $E_{0}=18574.8 \pm 0.6 \mathrm{eV}$. From $E_{0}$ we calculate the mass difference

$m(\mathrm{~T})-m\left({ }^{3} \mathrm{He}\right)=18591 \pm 3 \mathrm{eV} / c^{2}$,

\#3 An admixture of a second neutrino in the range $m_{\nu}^{2} \leqslant$ $100(\mathrm{eV} / \mathrm{c})^{2}$ could remove neither the unphysical value of negative $m_{\nu}^{2}$ nor the trends in $m_{\nu}^{2}$ and $E_{0}$ with increasing data set. In contrast to additional shake off components the inclusion of a second neutrino does not lead to the observed spectral shape due to its essentially different functional dependence.

\#4 Extrapolated towards lower energies this fit yields progressively less count rate than measured. When we plot the cube root of this excess count rate (like in fig. 2), a nice, straight Kurie line shows up which intersects with the abscissa $75 \mathrm{eV}$ below the endpoint. Thus it has the signature of a missing spectral component with that endpoint and an amplitude of $4 \%$. We note that the centre of gravity of the shake off electrons is $69 \mathrm{eV}$.
Table 2

Results for $m_{\nu}^{2}, E_{0}$ and $\chi^{2} / D O F$ for the two sources S1, S2 and the combined fit $\Sigma$.

\begin{tabular}{llll}
\hline Source & $\begin{array}{l}m_{\nu}^{2} \pm \Delta m_{\nu}^{2} \\
\left((\mathrm{eV})^{2} / c^{4}\right)\end{array}$ & $\begin{array}{l}E_{0} \pm \Delta E \\
(\mathrm{eV})\end{array}$ & $\chi^{2} / \mathrm{DOF}$ \\
\hline S1 & $-46 \pm 56$ & $18574.2 \pm 0.7$ & 0.93 \\
S2 & $-29 \pm 43$ & $18575.3 \pm 0.8$ & 0.93 \\
$\Sigma$ & $-39 \pm 34$ & $18574.8 \pm 0.6$ & 0.70 \\
\hline
\end{tabular}

where the following corrections have been taken into account: recoil energy $(1.7 \mathrm{eV})$, difference in chemical binding energies $(16.5 \mathrm{eV})$, polarization shift $(-0.9$ eV) [14], difference in work functions between substrate and analyzing electrode $(-0.1 \mathrm{eV})$ [12], potential drop in the analyzing plane $(-1.2 \mathrm{eV})[11]$. The error is dominated by the uncertainty in the high voltage measurement [12]. Our measurement of the mass difference matches well with recent results $[6,7,26]$.

Following the recipe of the Particle Data Group [27] we calculate from our $m_{\nu}^{2}$ result the following upper limit for the electron-antineutrino rest mass with $95 \%$ confidence level:

$m_{\nu}<7: 2 \mathrm{eV} / \mathrm{c}^{2}$

In table 3 we have listed recent measurements of $m_{\nu}^{2}$. 
Table 3

Recent results of $m_{\nu}^{2}$ from tritium $\beta$-decay. Values of $\sigma_{\text {stat }}$ and $\sigma_{\text {syst }}$ are $1 \sigma$ errors. Upper limits on $m_{\nu}$ according to ref. [27] correspond to $95 \% \mathrm{CL}$.

\begin{tabular}{lcl}
\hline Reference & $\begin{array}{l}m_{\nu}^{2} \pm \sigma_{\text {stat }} \pm \sigma_{\text {syst }} \\
\left((\mathrm{eV})^{2} / \mathrm{c}^{4}\right)\end{array}$ & $\begin{array}{l}m_{\nu} \\
\left(\mathrm{eV} / \mathrm{c}^{2}\right)\end{array}$ \\
\hline LANL [6] & $-147 \pm 68 \pm 41$ & $<9.3$ \\
Zürich [7] & $-24 \pm 48 \pm 61$ & $<11$ \\
INS [28] & $-65 \pm 85 \pm 65$ & $<13$ \\
LLNL [29] & $-72 \pm 41 \pm 30$ & $<8$ \\
this paper & $-39 \pm 34 \pm 15$ & $<7.2$ \\
\hline
\end{tabular}

Among the known sources of the systematic error of the present result, uncertainties in the energy loss fraction and the backscatter from the substrate dominate. These values will be checked in detail by measureménts with electron conversion lines from ${ }^{83 \mathrm{~m}} \mathrm{Kr}$ covered with $\mathrm{D}_{2}$ layers of known thickness in the near future. The remaining problem of not fully understanding the measured $\beta$-spectrum may be circumvented by restricting the analysis to a region very close to the endpoint which may be even smaller than the one used here. The unique capability of working very close to the endpoint has not been fully exploited in the past. To this end we will considerably improve the statistical accuracy and make an effort to further reduce background.

The spectrometer was financed by the state of Rheinland-Pfalz and the Bundesminister für Bildung und Wissenschaft providing funds for the new Physics building of the University and its equipment. The Deutsche Forschungsgemeinschaft has contributed to the running and personnel costs of the experiment under the contract number OT33-11. We thank V.M. Lobashev for critical discussions while writing this paper. One of us (R.B.M.) acknowledges a NATO collaborative grant for support of this work.

\section{References}

[1] V.A. Lubimov, E.G. Novikov, V.Z. Nozik, E.F. Tretyakov and V.S. Kosik, Phys. Lett. B 94 (1980) 266.

[2] S. Boris, A. Golutvin, L. Laptin, V. Lubimov, V. Nagovizin, V. Nozik, E. Novikov, V. Soloshenko, I. Tihomirov, E. Tretyakov and N. Myasoedov, Phys. Rev. Lett. 58 (1987) 2019.
[3] E.F. Tretyakov, Bull. USSR Acad. Sci. Phys., Ser. 39 , No. 3 (1975) 102.

[4] M. Fritschi, E. Holzschuh, W. Kuendig, J.W. Petersen, R.E. Pixley and H. Stuessi, Phys. Lett. B 173 (1986) 485.

[5] J.F. Wilkerson, T.J. Bowles, J.C. Browne, M.P. Maley, R.G.H. Robertson, J.S. Cohen, R.L. Martin, D.A. Knapp and J.A. Helffrich, Phys. Rev. Lett. 58 (1987) 2023

[6] R.G.H. Robertson, T.J. Bowles, G.J. Stephenson, D.L. Wark, J.F. Wilkerson and D.A. Knapp, Phys. Rev. Lett. 67 (1991) 957.

[7] E. Holzschuh, M. Fritschi and W. Kündig, Phys. Lett. B 287 (1992) 381.

[8] V.M. Lobashev, A.I. Fedoseyev, D.V. Serdyuk and A.P. Solodukhin, Nucl. Instrum. Methods A 240 (1985) 305.

[9] H. Backe, J. Bonn, Th. Edling, H. Fischer, A. Hermanni, P. Leiderer, Th. Loeken, R.B. Moore, A. Osipowicz, E.W. Otten and A. Picard, Phys. Scr. T 22 (1988) 98.

[10] S. Balashov et al., Proc. II Intern. Symp. on Weak and electromagnetic interactions in nuclei (WEIN89) (Montreal, 1989) (Editions Frontières, Gif-surYvette, France) p. 295.

[11] A. Picard, H. Backe, H. Barth, J. Bonn, B. Degen, Th. Edling, R. Haid, A. Hermanni, P. Leiderer, Th. Loeken, A. Molz, R.B. Moore, A. Osipowicz, E.W. Otten, M. Przyrembel, M. Schrader, M. Steininger and Ch. Weinheimer, Nucl. Instrum. Methods B 63 (1992) 345.

[12] A. Picard, H. Backe, J. Bonn, B. Degen, R. Haid, A. Hermanni, P. Leiderer, A. Osipowicz, E.W. Otten, M. Przyrembel, M. Schrader,M. Steininger and Ch. Weinheimer, Z. Phys. A 342 (1992) 71.

[13] Ch. Weinheimer, M. Schrader, J. Bonn, Th. Loeken and H. Backe, Nucl. Instrum. Methods A 311 (1992) 273.

[14] W. Kolos, B. Jeziorski, J. Rychlewski, K. Szalewicz, H.J. Monkhorșt and O. Fackler, Phys. Rev. A 37 (1988) 2297.

[15] O. Fackler, B. Jezior'ski, W. Kolos, H.J. Monkhorst and K. Szalewicz, Phys. Rev. Lett. 55 (1985) 1388.

[16] M. Przyrembel, H. Fischer, A. Hermanni, E.W. Otten and P. Leiderer, Phys. Lett. A 147 (1990) 517.

[17]. J. Geiger, Z. Phys. 181 (1964) 413.

[18] A.E.S. Green and T. Sawada, J. Atm. Terr. Phys. 34 (1972) 1719.

[19] L. Pages, E. Bertel, H. Joffre and L. Skalventis, At. Data 4 (1972) 1.

[20] J.W. Liu, Phys. Rev. A 7 (1973) 103.

[21] J.J. Simpson, Phys. Rev. D 23 (1981) 649.

[22] L. Martin et al., Phys. Lett. A 110 (1985) 95.

[23] D.A. Knapp, doctoral thesis, LANL (Los Alamos, 1986).

[24] D.L. Wark, R. Bartlett, T.J. Bowles, A.G.H. Robertson, D.S. Sivia, W. Trela, J.F. Wilkerson, G.S. Brown, 
B. Crasemann, S.L. Sorensen, S.J. Schaphorst, D.A. Knapp, J. Henderson, J. Tulkki and T. Åberg, Phys. Rev. Lett. 67 (1991) 2291.

[25] Th.A. Carlson and C.W. Nestor, Phys. Rev. A 8 (1973) 2887.

[26] R.S. Van Dyck, D.L. Farnham, J. Bare and P.B. Schwinberg, Proc. sixth Intern. Conf. on Nuclei far from stability and ninth Intern. Conf. on Atomic masses and fundamental constants (Bernkastel-Kues, 1992), eds. K.L. Kratz et al., IOP Conf. Series (IOP, Bristol), in print.
[27] Particle Data Group, G.P. Yost et al., Review of particle properties, Phys. Lett. B 204 (1988) 69.

[28] H. Kawakami, S. Kato, T. Ohshima, S. Shibata, K. Ukai, N. Morikawa, N. Nogawa, K. Haga, T. Nagafuchi, M. Shigeta, Y. Fukushima and T. Taniguchi, Phys. Lett. B 256 (1991) 105.

[29] W. Stoeffl, Bull. Am. Phys. Soc. 37, No. 5 (1992) 1286. 